Cahiers de la recherche sur les droits fondamentaux

Recherche Fon les Droits

$17 \mid 2019$

La motivation des actes administratifs. Le droit français à la lumière du droit administratif comparé

\title{
La motivation des actes administratifs locaux : entre protection de l'administré et intérêt public local
}

Annie Fitte-Duval

(2) OpenEdition

Journals

Édition électronique

URL : https://journals.openedition.org/crdf/3645

DOI : $10.4000 /$ crdf.3645

ISSN : 2264-1246

Éditeur

Presses universitaires de Caen

Édition imprimée

Date de publication : 31 décembre 2019

Pagination : 11-20

ISBN : 978-2-84133-960-0

ISSN : $1634-8842$

Référence électronique

Annie Fitte-Duval, «La motivation des actes administratifs locaux : entre protection de l'administré et

intérêt public local », Cahiers de la recherche sur les droits fondamentaux [En ligne], 17 | 2019, mis en

ligne le 06 février 2021, consulté le 14 novembre 2022. URL : http://journals.openedition.org/crdf/3645

; DOI : https://doi.org/10.4000/crdf.3645 


\title{
La motivation des actes administratifs locaux: entre protection de l'administré et intérêt public local
}

\author{
Annie FITTE-DUVAL \\ Maîtresse de conférences (HDR) en droit public à l'université de Pau et des Pays de l'Adour \\ Laboratoire Pau droit public (PDP)
}

I. La protection de l'administré local au cœur d'une motivation sélective

A. Une motivation centrée sur les décisions défavorables

B. Portée et limites de la définition retenue

1. La prise en considération du seul destinataire

2. Les exclusions liées à la nature des actes

3. Les exclusions liées à la reconnaissance d'un pouvoir étendu à l'administration

II. La motivation sélective, contrariée par la spécificité des enjeux locaux
A. La diversification des sources, facteur d'élargissement de l'obligation de motivation
1. L'articulation des différentes règles de motivation
2. L'élargissement de la motivation à certains actes réglementaires
3. La motivation par référence
B. La motivation des actes locaux, une fonction ambivalente au regard de la libre administration et de l'intérêt public local

\begin{abstract}
À l'heure de la transparence numérique dans un cadre supranational, les collectivités territoriales doivent de plus en plus justifier leurs décisions, tant sur le fond que dans la forme. Traditionnellement pourtant, les décisions de l'administration ne sont soumises qu'à de rares conditions de forme. La motivation de certains actes administratifs en fait partie et les collectivités territoriales n'échappent pas aux règles générales définies en la matière. Le principe de la motivation de certaines décisions administratives est posé dans l'article 211-2 du Code des relations entre le public et l'administration (CRPA) qui a opéré une codification à droit constant ${ }^{1}$, et reprend sur ce point la loi du 11 juillet 1979 relative à la motivation des actes administratifs.
\end{abstract}

Les articles L. 211-2 et L. 211-3 du Code réitèrent le principe de la motivation facultative, à l'exception de certaines catégories d'actes administratifs scrupuleusement énumérées, conservant ainsi une tradition française rétive à la généralisation de la motivation. À la suite du Conseil d'État ${ }^{2}$, le Conseil constitutionnel reconnaît l'absence de caractère obligatoire de la motivation, en précisant

$[\ldots]$ que les règles et principes de valeur constitutionnelle n'imposent pas par eux-mêmes aux autorités administratives de motiver leurs décisions dès lors qu'elles ne prononcent pas une sanction ayant le caractère d'une punition $^{3}$.

1. Cette codification intervient conformément à l'article 3 de la loi du 12 novembre 2013 habilitant le gouvernement à simplifier les relations entre l'administration et les citoyens.

2. CE, sect., 26 janvier 1973, Ministre de la Justice c. Sieur Lang, n 87890, Recueil Lebon, p. 72.

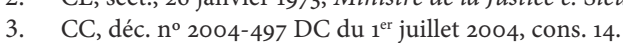


Avec pragmatisme, la doctrine avait fourni dès l'adoption de la loi du 11 juillet 1979 des explications pertinentes à ce choix français soulignant qu'à l'évidence, l'obligation de motivation ne saurait affecter l'ensemble des actes administratifs, non seulement parce que la procédure administrative s'en trouverait ralentie mais aussi parce qu'il était important

[...] de ne pas enserrer l'activité administrative dans un carcan d'obligations trop strictes, de ne pas alourdir la procédure d'élaboration des décisions, de ne pas multiplier les occasions d'annulation pour vice de forme ${ }^{4}$.

Une autre raison justifiant la sélectivité du principe de motivation pourrait résider dans la spécificité de la conception française de l'intérêt général, traditionnellement en surplomb des intérêts particuliers antagonistes et dont la définition incombe aux gouvernants. Or, à la faveur notamment d'une désacralisation visible de l'action publique et d'une exigence croissante de transparence, singulièrement valorisée par le droit européen, les modalités de définition de l'intérêt général évoluent, tenant parfois de la collision des intérêts particuliers, accentuant le nécessaire arbitrage d'administrateurs contraints d'assortir leurs décisions de justifications de plus en plus pointilleuses. Les rédacteurs de la loi de 1979 en étaient déjà conscients lorsqu'ils affirmaient dans l'exposé des motifs de la loi:

Les Français exigent maintenant de connaître les raisons des mesures prises à leur égard. Ils y voient le symbole d'un nouveau type de rapports entre l'État et eux, le moyen d'exercer un contrôle plus précis sur les actes administratifs, la promesse de réflexions plus attentives de l'administration au moment où elle prend des décisions qui leur sont opposables.

L'impartialité n'est pas seulement éthique des juges, elle s'impose de plus en plus à l'action publique en général et à l'administration de plus en plus concernée par une théorie des apparences ${ }^{5}$ qui va bien au-delà de sa source première, l'action du juge. Tous les actes administratifs reposent sur des motifs et l'absence de pertinence de ces motifs en droit ou en fait porte atteinte à la légalité interne de l'acte, entaché d'erreur de droit ou de fait. Ainsi, la cause est entendue, la motivation des actes administratifs est une obligation formelle, de celles qui peuvent être corrigées dès lors que

[...] l'illégalité externe [peut être définie] comme celle qui, une fois constatée, laisse intacte la faculté, pour l'administration, de refaire l'acte sans violer la chose jugée par le juge de l'excès de pouvoir. Au contraire, l'annulation pour illégalité interne peut être comprise comme celle qui, sauf exception, interdit à l'administration de reprendre la même décision ${ }^{6}$.

Néanmoins l'extension du principe de motivation résulte bien d'un mouvement de fond qui, loin de se limiter à une exigence technique, contribue à l'idée d'une démocratie administrative, laquelle suppose notamment que les droits procéduraux des administrés se rapprochent de véritables «droits-créances». Pour autant, ni la Convention européenne ni la Charte des droits fondamentaux, qui prévoient expressément des garanties procédurales et d'impartialité, ne font obligation aux États parties de motiver toutes leurs décisions. Certes, la Convention européenne de sauvegarde des droits de l'homme et des libertés fondamentales prévoit l'obligation de motivation pour les décisions qui entrent dans le champ de l'article 6 de la Convention européenne des droits de l'homme et le droit au procès équitable se nourrit partiellement mais inéluctablement de cette exigence, ce que la doctrine explique volontiers par le fait que plus l'administré est en mesure de connaître les motifs des décisions le concernant, plus il aura de possibilités de contester efficacement l'acte litigieux, et donc d'exercer son droit au recours.

Par ailleurs, certains textes du droit de l'Union prévoient la motivation des actes administratifs des États membres dans un domaine particulier et l'article 41 de la Charte des droits fondamentaux de l'Union européenne mentionne «l'obligation pour l'administration de motiver ses décisions». Même si cette dernière disposition ne vise que les institutions de l'Union, un tel parti pris textuel ne saurait être sans incidence sur le droit des États membres, clairement incités par là même à suivre l'exemple de la «bonne administration".

La France n'est pas tout à fait seule à avoir fait le choix de la sélectivité des décisions administratives soumises à l'obligation de motivation, mais la solution d'une contrainte légale et générale de motivation des actes de l'administration semble prédominer aujourd'hui au sein des pays européens ${ }^{7}$ tandis qu'en France, il faut bien le constater, la motivation dans les décisions administratives prend une place croissante.

Ce processus d'extension, identifiable avant même que la loi du 11 juillet 1979 n'ait été modifiée, s’inscrit également très nettement dans l'action locale ${ }^{8}$. Si la motivation des actes administratifs locaux ne revêt pas davantage le caractère d'une obligation générale, les sources qui mettent à

4. CE, Ass., 27 novembre 1970, Agence maritime Marseille Frêt, n 74877, 75123, concl. M. Gentot, Recueil Lebon, p. 704; Revue du droit public, 1971, p. 987.

5. A. Fitte-Duval, «La théorie des apparences, nouveau paradigme de l'action publique?», L'actualité juridique. Droit administratif, 5 mars 2018 , p. 440 sq.

6. CE, sect., 23 mars 1956, Dame Veuve Ginestet, no 3587 , concl. J. Kahn.

7. Voir par exemple I. Papadamaki, «L'obligation de motivation en droit administratif français sous l'influence du droit de l'Union européenne», Revue du droit public, 2017, p. 1245 sq.

8. La loi n'a connu que deux modifications depuis son adoption: la première, avec la loi no $86-76$ du 17 janvier 1986, a essentiellement eu pour objet d'étendre la motivation obligatoire aux décisions refusant des autorisations ainsi qu'aux décisions des organismes de Sécurité sociale et d'assurance chômage refusant des prestations facultatives. La deuxième, avec la loi n 2011-525 du 17 mai 2011, a étendu la motivation obligatoire aux décisions administratives rejetant un recours administratif préalable obligatoire. 
la charge des collectivités une obligation de motivation se multiplient. Le Conseil constitutionnel a jugé que seul le législateur pouvait instaurer, dans le cas des collectivités territoriales, l'obligation de motiver les décisions, cette obligation touchant aux principes fondamentaux de la libre administration de ces collectivités. Le principe de libre administration ayant pour corollaire le contrôle de légalité, la question de la motivation des décisions locales se révèle étroitement dépendante d'un arbitrage convoquant libre administration, contrôle de légalité, intérêt local et intérêt de l'administré.

Si la motivation de la plupart des décisions locales relève de l'obligation générale posée par la loi de 1979, d'autres obligations de motiver particulières sont instituées par des sources spéciales. Le droit des collectivités territoriales est en effet un lieu de rencontre entre les règles spécifiques créées pour les collectivités et les normes générales qui s'appliquent à elles. Ainsi, il n'est pas tout entier contenu dans le Code général des collectivités territoriales et les collectivités doivent aussi tenir compte de prescriptions contenues dans d'autres textes, comme le Code de l'urbanisme ou celui des marchés publics, ou le statut de la fonction publique. L'encadrement de la procédure administrative non contentieuse applicable dans les collectivités territoriales requiert notamment l'application combinée du CRPA et du Code général des collectivités territoriales même si les domaines de ces deux codes demeurent distincts.

L'énumération des décisions concernées par l'obligation de motivation désormais énoncée par l'article L. 211-2 du CRPA s'applique sans restriction aux relations des collectivités avec leurs administrés. La motivation, lorsqu'elle est obligatoire, participe de la régularité de la décision administrative et relève du contrôle de sa légalité externe; aussi le juge reconnaît-il la pertinence d'une « obligation de motivation en la forme $»^{9}$.

Le principe du caractère facultatif de la motivation se traduit notamment par l'affirmation traditionnelle selon laquelle il n'y a "pas de motivation sans texte» : le Conseil d'État avait ainsi conclu à l'absence d'obligation de motiver les actes de police en Polynésie, faute que la loi du 11 juillet 1979 y ait été rendue applicable ${ }^{10}$. Le Conseil constitutionnel a, en revanche, plus récemment jugé que les règles relatives à la motivation des actes administratifs relèvent des droits des citoyens dans leurs relations avec l'administration - et qu'en tant qu'elle porte sur les actes administratifs des administrations de l'État et des communes ou de leurs établissements publics, la loi du 11 juillet 1979 est applicable de plein droit en Polynésie française sans qu'il soit nécessaire qu'une disposition législative le précise ${ }^{11}$.

Mais la motivation n'est pas qu'exigence formelle. Outre l'étroite corrélation qui existe entre l'exigence de motivation et le contrôle juridictionnel des motifs de l'acte, la motivation, en facilitant l'information de l'administré, garantit également la qualité de l'échange entre l'administrateur et celui-ci, assoit la légitimité des décisions administratives et participe de la transparence et de la démocratie administrative. Plus que jamais, le droit de «demander compte à tout agent public de son administration » énoncé par l'article 15 de la Déclaration des droits de l'homme et du citoyen de 1789 prend tout son sens. Il devient de plus en plus difficile de ne voir dans la motivation qu'une simple modalité technique facilitant la relation administrative et d'en occulter la contribution à une forme de démocratie administrative. L'influence des textes européens et, partant, des institutions européennes, met en lumière la diversité des fonctions de la motivation, qui participe à la protection des droits fondamentaux des administrés. Elle suggère également le réexamen d'une solution bâtarde y compris dans les collectivités, qui est celle d'une motivation facultative assortie de dérogations multiples ${ }^{12}$.

Ainsi, la protection de l'administré local justifie a priori le périmètre d'une motivation obligatoire sélective (I) mais les exigences de motivation prévues par les textes spéciaux fragilisent un principe également malmené par la spécificité des enjeux locaux (II).

\section{La protection de l'administré local au cœur d'une motivation sélective}

L'exigence de transparence croissante conduit aussi dans la sphère décisionnelle locale à multiplier les hypothèses de motivation obligatoire sans remettre en question le principe d'une énumération limitative posé par le législateur.

Dès lors que le juge dans le cadre de l'instruction a toujours les moyens de contraindre l'administration à lui communiquer les motifs de son intervention, le principal bénéficiaire de la motivation obligatoire est l'administrécitoyen, et le citoyen local doit bénéficier d'une protection équivalente à celle accordée au national. La protection se concentre sur les actes défavorables (A) ce qui limite la portée de l'obligation (B).

\section{A. Une motivation centrée sur les décisions défavorables}

La pièce maîtresse du dispositif de motivation protecteur réside comme pour l'ensemble des collectivités publiques dans la contrainte issue de la loi du 11 juillet 1979 et codifiée à l'article L. 211-2 du CRPA qui énumère les décisions concernées par l'obligation. L'essentiel des décisions qui

9. CE, Ass., 5 novembre 2004, Union des organisations de fonctionnaires et assimilés, groupe des dix solidaires, $\mathrm{n}^{\circ} 252102$.

10. CE, 12 mai 1989, Ministre des départements et territoires d'outre-mer c. Piermont, $\mathrm{n}^{\circ}$ 85852, JurisData, $\mathrm{n}^{\circ}$ 1989-641847.

11. CC, déc. $n^{\circ}$ 2014-4 LOM du 19 septembre 2014, Motivation des actes administratifs en Polynésie française.

12. Voir, sur la question de la généralisation, O. Gabarda, «Vers la généralisation de la motivation obligatoire des actes administratifs? Enjeux et perspectives d'évolutions autour du principe de la motivation facultative», Revue française du droit administratif, 2012, p. 61 sq. 
font l'objet d'une motivation sélective sont des décisions défavorables ${ }^{13}$, même si l'article 211-3 exige également la motivation des décisions individuelles qui dérogent aux lois et règlements.

La circulaire du 2 juin 1992 relative à l'application aux collectivités territoriales de la loi $n^{\circ} 79-587$ du 11 juillet 1979 souligne la nécessité d'informer les élus au sujet des obligations qui leur incombent à cet égard et fournit, en annexe, à titre indicatif, une liste des décisions de compétence territoriale qui paraissent devoir être motivées.

Nombreuses sont les décisions des collectivités locales qui entrent dans l'une des catégories de décisions défavorables limitativement énumérées par le Code dans cet article et la jurisprudence administrative fournit pour chacune des catégories d'actes envisagées des illustrations de la contrainte de motivation subie par les collectivités territoriales au titre des catégories énumérées.

La liste initiale prévue par la loi de 1979 étant principalement constituée par des actes qui se définissent par leur caractère individuel et défavorable, il convient de préciser cette exigence. Alors qu'il suffit qu'un acte ne présente pas le caractère d'une décision individuelle pour que les dispositions de la loi ne lui soient pas applicables, le législateur énumère précisément les sept catégories de décisions défavorables qui doivent faire l'objet d'une motivation obligatoire ${ }^{14}$. Celles-ci renvoient à des champs d'intervention particulièrement signifiants pour les collectivités dans des domaines clés de leur activité que l'on ne saurait ici aborder de manière exhaustive. Nous nous bornerons à en évoquer quelques illustrations fortes, qui parfois relèvent de plusieurs rubriques de l'article L. 211-2, non sans avoir rappelé que la maîtrise du sol, les sanctions disciplinaires dans la fonction publique, les décisions d'internement dans un hôpital psychiatrique, les mesures de police font partie des hypothèses historiques de motivation (antérieures à la loi de 1979). $\mathrm{Si}$, sur le plan général, l'évolution des règles de transparence et de la communication entre l'administration et les citoyens a contribué à combler partiellement l'écart entre l'administration et les usagers, la proximité et la personnalisation sont des caractères structurels des relations entre l'administré et les collectivités, ce qui rend encore plus nécessaire le recours à la motivation des décisions défavorables. Leur énumération se révèle en effet signifiante pour les collectivités.
Aux termes de l'article L. 211-2, sont concernées en premier lieu les décisions «qui restreignent l'exercice des libertés publiques ou [...] constituent une mesure de police».

La fonction de police des maires fournit de nombreuses illustrations de cette première catégorie, qu'il s'agisse de la police générale, ou même des polices spéciales qui contribuent l'une et l'autre à l'ouverture d'un champ essentiel de l'action municipale. Les décisions administratives individuelles de police ayant un caractère défavorable entrent, par l'effet de l'article L. 211-2, dans le cadre des actes soumis à une obligation de motivation (en dehors des cas d'urgence absolue ou des décisions implicites, par exemple un refus implicite d'autorisation de police). À titre d'illustration, l'arrêté d'un maire ordonnant, sur le fondement de l'article L. 344 du Code de la santé publique, l'internement provisoire d'une personne atteinte d'aliénation mentale doit être motivé ${ }^{15}$. Le refus d'autorisation d'un spectacle ${ }^{16}$, la fermeture d'un restaurant ou d'un débit de boissons ${ }^{17}$ sont autant de mesures qui appellent une motivation en application de cette première catégorie de décisions défavorables recensée par l'article $1^{\text {er }}$ de la loi de 1979. Le maire n'est d'ailleurs pas la seule autorité locale disposant d'un pouvoir de police: ainsi, l'arrêté d'un président de conseil général prescrivant la fermeture d'un établissement d'hébergement de personnes âgées doit aussi faire l'objet d'une motivation ${ }^{18}$.

En second lieu, l'article 211-2 évoque les décisions qui infligent une sanction.

En matière de sanctions, les collectivités peuvent notamment être confrontées à celles qui touchent à l'agent, à l'usager ou au cocontractant. La sanction garantit le respect des droits de la défense et permet un contrôle de proportionnalité de la sanction à la faute. La gestion des agents publics fournit de nombreuses applications de la notion de décisions défavorables, au regard du pouvoir de sanction, en particulier dans l'utilisation du pouvoir disciplinaire. La sanction disciplinaire prononcée à l'encontre d'un agent public doit être motivée, et cette motivation doit être précise. Ainsi, un arrêté prononçant la révocation d'un fonctionnaire territorial à compter du 15 avril 2011 se bornant à mentionner qu'il est reproché à ce dernier d' «avoir manqué à l'obligation de dignité et de respect des agents », sans autre précision sur les circonstances de fait l'ayant motivé, ne satisfait pas à l'obligation de motivation

13. F. Colin, «L'acte administratif défavorable», Revue du droit public, 2018, p. 713 sq.

14. Art. L. 211-2: «Les personnes physiques ou morales ont le droit d’être informées sans délai des motifs des décisions administratives individuelles défavorables qui les concernent. / À cet effet, doivent être motivées les décisions qui : $/ 1^{\circ}$ Restreignent l'exercice des libertés publiques ou, de manière générale, constituent une mesure de police $; / 2^{\circ}$ Infligent une sanction; $/ 3^{\circ}$ Subordonnent l'octroi d'une autorisation à des conditions restrictives ou imposent des sujétions; $/ 4^{\circ}$ Retirent ou abrogent une décision créatrice de droits; $/ 5^{\circ}$ Opposent une prescription, une forclusion ou une déchéance; $/ 6^{\circ}$ Refusent un avantage dont l'attribution constitue un droit pour les personnes qui remplissent les conditions légales pour l'obtenir $; / 7^{\circ}$ Refusent une autorisation, sauf lorsque la communication des motifs pourrait être de nature à porter atteinte à l'un des secrets ou intérêts protégés par les dispositions du a au f du $2^{\circ}$ de l'article L. $311-5 ; / 8^{\circ}$ Rejettent un recours administratif dont la présentation est obligatoire préalablement à tout recours contentieux en application d'une disposition législative ou réglementaire. - [Loi n $79-587$ du 11 juillet 1979 , art. $\left.1^{\mathrm{er}}\right]$ ».

15. CE, 31 mars 1989, Ministre de l'Intérieur et de la Décentralisation et Lambert, $\mathrm{n}^{\circ} 69547$.

16. CE, 5 décembre $1984, S^{t e ́}$ Émeraude Show, no 49448.

17. CE, 13 février 1985, Bouamine, Droit administratif, 1985, comm. 116; CE, 26 novembre $2012, \mathrm{n}^{\circ} 344587$.

18. CE, 29 décembre 1995, $M^{\text {lle }}$ Cabrera, no 147685. 
prévue par les dispositions précitées, et cela, en dépit de la circonstance que l'agent a eu communication de l'avis du conseil de discipline, auquel l'arrêté se réfère sans toutefois le joindre ${ }^{19}$.

La gestion de carrière des agents publics révèle aussi un vivier important de décisions défavorables au titre des décisions qui « retirent ou abrogent une décision créatrice de droits ». Ainsi la motivation est obligatoire dans le cas d'une décision de fin de détachement sur emploi fonctionnel dans la fonction publique territoriale ${ }^{20}$. Le droit de la fonction publique locale peut d'ailleurs en la matière se révéler subtilement différent de celui de la fonction publique d'État. Ainsi, la collectivité territoriale de Corse ayant mis fin aux fonctions de directeur de l'agence de tourisme de la Corse s'est vue rappeler par la cour administrative d'appel de Marseille

[...] que si une décision mettant fin aux fonctions d'un agent occupant un emploi à la discrétion du gouvernement n'a pas à être motivée [...] le régime des emplois à la décision du gouvernement, fondé en dernier lieu sur les dispositions de l'article 25 de la loi no 84-16 du 11 janvier 1984 portant dispositions statutaires relatives à la fonction publique de l'État et non reprises par la loi du 26 janvier 1984 relative à la fonction publique territoriale, n'est susceptible de s'appliquer qu'à des agents employés par l'État ou des établissements publics qui en relèvent ${ }^{21}$

et donc ne permet pas d'écarter l'exigence de motivation dans ce cas de licenciement.

Sont également des décisions défavorables, celles qui «subordonnent l'octroi d'une autorisation à des conditions restrictives ou imposent des sujétions». La décision rejetant une demande présentée en vue d'obtenir une autorisation d'occupation du domaine public constitue un refus d'autorisation au sens des dispositions de l'article $1^{\text {er }}$ de la loi du 11 juillet 1979 relative à la motivation des actes administratifs et à l'amélioration des relations entre l'administration et le public, désormais codifié à l'article L. 211-2, $7^{\circ}$ du CRPA ${ }^{22}$.

La catégorie des décisions qui « refusent un avantage dont l'attribution constitue un droit pour les personnes qui remplissent les conditions légales pour l'obtenir» trouve aussi des applications, par exemple en droit de la fonction publique: le refus de réintégration d'un fonctionnaire territorial dans son administration à l'issue d'une disponibilité prononcée d'office à l'expiration des droits statutaires à congés de maladie ${ }^{23}$. Il appartient au juge d'identifier l'avantage constitutif d'un droit, comme le montre l'exemple suivant, l'article L. 5142-1 du Code général de la propriété des personnes publiques disposant que:

Dans le département de la Guyane, les immeubles domaniaux compris dans un plan d'occupation des sols opposable aux tiers, un plan d'urbanisme approuvé ou un document d'urbanisme en tenant lieu, peuvent faire l'objet: [...] $3^{\circ} \mathrm{De}$ cessions gratuites aux collectivités territoriales $[. .$.$] en vue$ de constituer sur le territoire d'une commune des réserves foncières dans les conditions fixées aux articles L. 221-1 et L. 221-2 du code de l'urbanisme [...].

Le Conseil d'État a jugé néanmoins que la cession par l'État d'une parcelle de son domaine privé n'est pas un droit pour celui qui en fait la demande et qu'une décision de refus de cession d'une telle parcelle n'est pas au nombre des décisions qui doivent être motivées en application de cet article ${ }^{24}$.

Les décisions qui "refusent une autorisation» sont sans doute celles qui offrent le plus de diversité dans des domaines éclectiques: en droit des services publics, le refus d'inscription d'un enfant dans une école communale constitue bien un refus d'autorisation ${ }^{25}$. Dans le domaine de la fonction publique, la décision par laquelle la commune refuse de faire droit à une demande de maintien en activité présentée en application de l'article 1.1 de la loi du 13 septembre 1984 relative à la limite d'âge dans la fonction publique et le secteur public (qui permet dans certaines conditions ce maintien) doit être regardée comme un refus d'autorisation, au sens de l'article $1^{\text {er }}$ de la loi du 11 juillet $1979^{26}$.

En matière d'urbanisme, le certificat d'urbanisme négatif doit être motivé aux termes de l'article R. 410-14 du Code de l'urbanisme et il sera illégal s'il n'indique pas notamment les dispositions d'urbanisme, les limitations administratives et la liste des participations applicable ${ }^{27}$.

En revanche, la décision de rejet d'une candidature dans le cadre d'une procédure de passation d'un contrat portant délégation de service public ne constitue pas le refus d'une autorisation au sens des dispositions de l'article $1^{\text {er }}$ de la loi du 11 juillet 1979 et n'entre dans aucune autre catégorie de décision administrative devant faire l'objet d'une motivation en application de ces dispositions ${ }^{28}$

L'article L. 211-2 ajoute aux décisions défavorables précédemment annoncées deux autres hypothèses de motivation. L'une qui relevait de l'article 2 de la loi de 1979 porte sur les décisions administratives individuelles qui dérogent aux règles générales fixées par la loi ou le règlement:

19. CAA Douai, $3^{\mathrm{e}} \mathrm{ch}$., formation à 3 (bis), 29 avril 2014, Commune de Verneuil-en-Halatte, $\mathrm{n}^{\circ}{ }_{13} \mathrm{DA} 01516$

20. CE, 3 mai 1993, M. Michel X., n 119805 et 119806.

21. CAA Marseille, 17 octobre 2013, M. Charavin, $\mathrm{n}^{\circ} 12 \mathrm{MA0} 3684$

22. CAA Bordeaux, 28 septembre 2017, $M^{m e}$ B et SARL Le Bikin, n ${ }^{\circ}{ }_{15}$ BXo 2360,15 BXo 2657 ; CAA Bordeaux, 15 novembre $2017, M$. D, n ${ }^{\circ} 15$ BXo 3680 .

23. Voir CE, 7 mai 2012, Commune de Roissy-en-France, $\mathrm{n}^{\circ} 346613$, tables du Recueil Lebon, p. 542.

24. CE, 24 octobre 2014, $M^{m e}$ A. B. c. commune de Remire-Montjoly, $\mathrm{n}^{\circ} 366966$, tables du Recueil Lebon.

25. CE, 10 mai 1996, Ville de Paris, $\mathrm{n}^{\circ}$ 136258, tables du Recueil Lebon, p. 682.

26. CE, 23 décembre 2011, $M^{\text {me }}$ Yvette A., n' 329016.

27. CAA Marseille, 2 mai 2013, $M^{\text {me }}$ Lang, $\mathrm{n}^{\circ} 11 \mathrm{MAoo} 86$.

28. CE, 27 janvier 2011, Commune de Ramatuelle, $\mathrm{n}^{\circ} 338285$, tables du Recueil Lebon. 
le droit administratif local connaît surtout de ces décisions dans le domaine de l'urbanisme. L'autre hypothèse désigne les décisions qui rejettent un recours administratif dont la présentation est obligatoire préalablement à tout recours contentieux (RAPO) ${ }^{29}$ : elles concernent notamment le domaine de la fonction publique, de l'urbanisme ou de l'aide sociale. On observe que les cas dans lesquels un recours administratif préalable est obligatoire concernent de plus en plus d'actes défavorables.

Ce tour d'horizon permet d'évaluer, sans souci d'exhaustivité, la diversité des décisions locales défavorables au sens de l'article 211-2. Leur portée et leurs limites doivent cependant être précisées.

\section{B. Portée et limites de la définition retenue}

En préalable, on observera que la méthode de délimitation des actes à motiver est sujette à discussion. Dès lors, en effet, que le législateur énumère de manière exhaustive des catégories, il prend le risque d'en oublier certaines. L'exemple de la discrimination est sur ce point édifiant: la loi no 2008-496 du 27 mai 2008 portant diverses dispositions d'adaptation au droit communautaire dans le domaine de la lutte contre les discriminations a fait le choix elle aussi (comme la loi $\mathrm{n}^{\circ} 79-587$ du 11 juillet 1979 sur la motivation des actes administratifs en son temps) d'établir une liste limitative des cas de discrimination. Et ce choix a conduit à une réécriture régulière du texte afin d'appréhender au plus juste les motifs de discrimination, ce qui induit une forme d'insécurité.

Même si l'énumération des décisions défavorables a peu évolué dans le temps, elle n'est pas pleinement satisfaisante. Certes, il était nécessaire de délimiter le champ des décisions défavorables, par essence complexe. Mais, outre le fait que l'énumération des actes défavorables ne suffit pas à lever toutes les incertitudes, puisqu'elle peut générer aussi des problèmes de définition internes à chaque rubrique, elle se révèle à l'usage discriminante, soit en raison de la prise en considération du seul destinataire (1), soit en raison de la nature des actes (2), soit en raison du pouvoir de l'administration (3).

\section{La prise en considération du seul destinataire}

Le caractère défavorable d'une décision s'évalue au regard des seules personnes physiques ou morales qui sont concernées par elle. Il ne suffit pas en effet que la décision occasionne un désagrément (éventuellement à d'autres personnes comme c'est le cas en ce qui concerne la demande d'une commune de se retirer d'un syndicat intercommunal) ou que l'agrément obtenu soit moins important que souhaité (exemple d'une décision accordant un permis de construire, même s'il est assorti de prescriptions spéciales).

Enfin, les décisions défavorables emportent des effets variables et parfois mitigés. Contraire aux intérêts de son destinataire, l'acte administratif défavorable peut à l'inverse profiter à des tiers, s'ils en tirent des avantages. Tel est le cas du refus d'une mesure de promotion dans la fonction publique qui peut bénéficier à un autre agent. En tout état de cause, il n'est pas rare qu'un acte administratif ait un double visage, et qu'il puisse en réalité être pour partie favorable et pour partie défavorable, déjà à son destinataire et a fortiori s'il existe des intérêts tiers.

\section{Les exclusions liées à la nature des actes}

Règle de forme, la motivation doit être écrite et reposer sur les considérations de fait et de droit qui fondent la décision. Les décisions implicites sont de ce fait, par leur nature même, dispensées de motivation. Sur demande de l'administré, la motivation devra lui en être communiquée dans le délai d'un mois. Le Conseil d'État refuse les formulations stéréotypées ${ }^{30}$. Cette exigence doit notamment permettre d'écarter les décisions arbitraires ou discriminatoires.

Certaines décisions de refus ou de rejet ne correspondent à aucune des hypothèses énoncées. Le juge doit parfois le constater: à titre d'exemple, le refus d'un maire de laisser un député accéder à des services municipaux non ouverts au public ${ }^{31}$ ne porte pas atteinte à l'exercice d'une liberté publique et n'a donc pas à être motivé.

L'acte réglementaire, à caractère général et impersonnel, est par nature un acte qui ne peut pas être qualifié d'acte défavorable au sens de l'article 211-2 puisqu'appliqué indifféremment à un ensemble de destinataires potentiels. À ce titre, la délibération d'un conseil municipal décidant la cession d'un ensemble immobilier appartenant à son domaine privé, à deux associations culturelle et socioculturelle ${ }^{32}$, tout comme la délibération d'un conseil municipal créant un emploi, en l'espèce, d'ouvrier d'entretien de la voie publique ${ }^{33}$, ne constituent pas des décisions défavorables.

La loi écarte de son champ d'application les actes collectifs et, par exemple, le refus d'établir un tableau d'avancement considéré comme « un acte collectif composé de plusieurs décisions à caractère individuel» n'est pas soumis à motivation au titre de la loi du 11 juillet $1979^{34}$.

29. L'institution du recours préalable obligatoire permet à l'autorité compétente de préciser la position de l'administration et la décision prise à la suite du recours, qui remplace la décision initiale, est seule susceptible d'être déférée au juge administratif (CE, sect., 18 novembre 2005, $H$., nº 270075). Cette solution conduit à écarter la recevabilité de toute contestation portée devant le juge sans décision administrative préalable au recours administratif obligatoire (CE, 25 janvier 2016, Ministre de la Défense c. H., n³ 387856) ou sans exercice de ce recours (CE, 6 juin 1977, Garrigues, nº 2962).

30. CE, 26 janvier 1968, Sté Maison Genestal, no 69765, Recueil Lebon, p. 62, concl. Bertrand.

31. CE, 18 octobre 1991, Rouquette, $\mathrm{n}^{\circ}$ 56635, Recueil Lebon, p. 340 ; La semaine juridique, édition générale, $\mathrm{n}^{\circ} 3,15$ janvier $1992, \mathrm{IV}, \mathrm{p} .13$.

32. CE, 25 novembre 2009, Commune de Mer, no 310208 .

33. CE, 9 novembre 1983, Lainé, $\mathrm{n}^{\circ} 40101$.

34. CE, 21 mars 2001, Syndicat lutte pénitentiaire Union régionale Antilles-Guyane, Recueil Lebon, p. 144. 
Les actes sui generis ne sont pas davantage soumis à l'obligation prévue par la loi de 1979: tel est le cas des déclarations d'expropriation d'utilité publique (dont on verra qu'elles relèvent en réalité d'une autre forme de motivation).

Insusceptibles de faire l'objet d'un recours pour excès de pouvoir, en raison de leur caractère non décisoire, les mesures d'ordre intérieur, dès lors qu'elles ne traduisent aucune discrimination, ne sont pas considérées comme défavorables en dépit des conséquences désavantageuses pour leur destinataire: ces mesures

[...] tout en modifiant leur affectation ou les tâches qu'ils ont à accomplir, ne portent pas atteinte aux droits et prérogatives qu'ils tiennent de leur statut ou à l'exercice de leurs droits et libertés fondamentaux, ni n'emportent perte de responsabilités ou de rémunération [...]; le recours contre de telles mesures, à moins qu'elles ne traduisent une discrimination, est irrecevable ${ }^{35}$.

\section{Les exclusions liées à la reconnaissance d'un pouvoir étendu à l'administration}

En dépit de la richesse de l'énumération des décisions défavorables, certaines décisions perçues comme défavorables par leurs destinataires demeurent exclues du champ défini par l'article L. 211-2 du CRPA, faute de pouvoir les rattacher à une des hypothèses prévues. Il en est ainsi des décisions discrétionnaires, qui laissent une marge d'appréciation importante à leur auteur comme les délibérations d'un jury de concours ou d'examen ${ }^{36}$ ou les notes attribuées aux candidats à un concours ${ }^{37}$.

La reconnaissance de l'acte défavorable au sens de l'article 211-2 se trouve également exclue du fait de la précarité de la situation du destinataire dans l'incapacité de se prévaloir d'un acte créateur de droits. Le licenciement du stagiaire en fin de stage n'impose pas de motivation, faute pour l'agent concerné de bénéficier d'un droit à la titularisation. En revanche, la nécessité de protéger un secret ou un intérêt protégé se retrouve plus rarement dans le cadre de l'action locale, certes assujettie au respect du secret des personnes mais moins soumise au secret qui protège la défense ou la sécurité.

L'article L. 211-1 permet de soumettre à l'obligation de motivation une part importante de l'activité des collectivités, mais ne régit pas ou seulement partiellement les actes pris dans certains secteurs qui présentent des enjeux spécifiques pour leur liberté d'action. Aussi, le législateur a-t-il également fait de la motivation, tant à travers la diversification des textes que des formes, un outil de vigilance concernant l'action locale. Dès lors, le principe de la motivation sélective s'avère contrarié par les enjeux locaux.

\section{La motivation sélective, contrariée par la spécificité des enjeux locaux}

Comme cela a pu déjà être souligné, les lois générales de procédure administrative, et, en leur sein, les dispositions du CRPA issues de la loi de 1979 s'appliquent aux administrations locales, en principe, mais doivent prendre en compte les spécificités et les enjeux propres de celles-ci. Le cadre étroit d'une motivation sélective retenu par le législateur dans la loi du 11 juillet 1979 craque sous l'impulsion de sources de plus en plus diversifiées de l'obligation de motivation (A), qui témoignent d'une ambivalence des enjeux liés à la libre administration des collectivités et à la détermination de l'intérêt public local (B).

\section{A. La diversification des sources, facteur d'élargissement de l'obligation de motivation}

La relation entre des sources différentes d'obligation de motivation peut être complexe.

\section{L'articulation des différentes règles de motivation}

Les règles spécifiques aux collectivités peuvent être prévues par des textes généraux. Ainsi le Code général des collectivités territoriales prévoit-il plusieurs hypothèses de motivation d'actes administratifs locaux. Mais, des textes spéciaux interviennent également dans des domaines qui ne sont pas - ou seulement partiellement - abordés par le CRPA, tels que l'urbanisme, la passation des contrats, la maîtrise des sols, qui renvoient à la diversité des compétences locales.

Ces règles de motivation peuvent être liées à l'article 211-2 du CRPA, ou relever de sources autonomes. À titre d'illustration de la concordance du texte général et spécial, par exemple, la suspension de l'agrément d'un assistant maternel ou familial constitue une mesure de police administrative prise dans l'intérêt des enfants accueillis par le président du conseil départemental. Elle doit être motivée en vertu des dispositions spéciales de l'article L. 421-6 du Code de l'action sociale et des familles, mais n'en relève pas moins du champ d'application de l'article $1^{\text {er }}$ de la loi $\mathrm{n}^{\circ} 79-587$ du 11 juillet 1979, désormais codifié à l'article L. 211-2 du CRPA ${ }^{38}$.

D'autres textes prévoient, en revanche, des obligations spéciales de motivation à la charge des collectivités qui ne sont pas tenues de respecter les critères fixés par le CRPA. Ainsi, par exemple, le Code des marchés publics (article 83) prévoit-il que le rejet de l'offre d'un candidat évincé doit être communiqué en en indiquant les motifs ${ }^{39}$. 
De même, la déclaration de projet d'intérêt général est une décision non réglementaire qui échappe à la loi de 1979, elle n'en doit pas moins être motivée au titre de l'article L. 126-1 du Code de l'environnement ${ }^{40}$.

La tendance, qui n'a fait que croître à la faveur de la diversification statutaire des collectivités et groupements territoriaux, peut être illustrée par un double mouvement: l'élargissement de la motivation à certains actes réglementaires, d'une part, et le développement de la motivation par référence, d'autre part.

\section{L'élargissement de la motivation à certains actes réglementaires}

En principe, les collectivités territoriales n'ont pas à motiver les actes réglementaires. Il en va différemment si un texte spécial le prévoit. Tel est le cas des articles L. 2213-2 à L. 2213-5 du Code général des collectivités territoriales en matière de police de la circulation et singulièrement en matière de circulation et de stationnement sur les voies publiques. Les articles L. 2213-2 à L. 2213-5 énoncent différentes interdictions et restrictions soumises à l'obligation de motivation: interdiction d'accès de certaines voies à certaines heures ou à certains véhicules, réglementation $\mathrm{du}$ stationnement ou du transport de marchandises. Les actes réglementaires pris par le maire sur ce fondement doivent comporter l'exposé des motifs de fait qui ont conduit le maire à adopter sa réglementation et sa base juridique. L'absence de telles précisions conduit le Conseil d'État à annuler l'arrêté:

Considérant [...] que pour motiver son arrêté du 21 mai 1991, le maire de Terre-de-Haut s'est borné à citer le code des communes «notamment les articles L. 131-3 et L. 131-4"; qu'il n'a pas précisé les circonstances de fait justifiant l'application à la commune de Terre-de-Haut d'une interdiction de la circulation des véhicules à moteurs terrestres de $9 \mathrm{~h}$ à $12 \mathrm{~h}$ et de $14 \mathrm{~h}$ à $16 \mathrm{~h}$; que dès lors, le maire n'a pas satisfait à l'obligation de motiver sa décision qui lui était faite par l'article L. 131-4 du code des communes précité; qu'ainsi les requérants sont fondés à soutenir que c'est à tort que, par le jugement attaqué, le tribunal administratif de Basse-Terre a refusé d'annuler cette décision $[\ldots]^{41}$.

La nécessité de motivation va parfois au-delà de la stricte prise en compte des exigences de la circulation: ainsi, compte tenu des termes de l'article L. 2213-2 du Code général des collectivités territoriales, un arrêté municipal réglementant le stationnement payant doit être motivé, non seulement par référence aux nécessités de la circulation, mais également eu égard à la protection de l'environnement ${ }^{42}$. Ainsi, par arrêté motivé, le maire peut,

[...] eu égard aux nécessités de la circulation et de la protection de l'environnement $[\ldots]$ interdire à certaines heures l'accès de certaines voies de l'agglomération ou de certaines portions de voie ou réserver cet accès, à certaines heures, à diverses catégories d'usagers et de véhicules [...]. (art. L. 2213-2)

$\mathrm{Ou}$

[...] interdire l'accès de certaines voies ou de certaines portions de voies ou de certains secteurs de la commune aux véhicules dont la circulation sur ces voies ou dans ces secteurs est de nature à compromettre soit la tranquillité publique, soit la qualité de l'air, soit la protection des espèces animales ou végétales, soit la protection des espaces naturels, des paysages ou des sites ou leur mise en valeur à des fins esthétiques, écologiques, agricoles, forestières ou touristiques. (art. L. 2213-4)

La loi peut en outre autoriser une motivation par référence.

\section{La motivation par référence}

Plusieurs cas de figure peuvent être identifiés.

En ce qui concerne les décisions de préemption, la loi solidarité et renouvellement urbains du 13 décembre 2000 a ajouté à l'article L. 210-1 du Code de l'urbanisme un alinéa aux termes duquel:

Lorsque la commune a délibéré pour définir le cadre des actions qu'elle entend mettre en ouvre pour mener à bien un programme local de l'habitat [...], la décision de préemption peut, sauf lorsqu'il s'agit d'un bien mentionné à l'article L. 211-4, se référer aux dispositions de cette délibération.

La collectivité a donc le choix entre la motivation classique dans l'acte et la motivation par référence à la délibération, mais l'assouplissement ne porte que sur les modalités d'inscription de la motivation qui demeure soumise à la même exigence de précision dans l'information donnée.

Le formalisme propre à la motivation traditionnelle cède aussi en matière de déclaration d'utilité publique. Ainsi, l'article L. 11-1-1 du Code de l'expropriation pour cause d'utilité publique, issu de la loi $\mathrm{n}^{\circ}$ 2002-276 du 27 février 2002, relative à la démocratie de proximité, dispose que «[1]'acte déclarant l'utilité publique est accompagné d'un document qui expose les motifs et considérations justifiant le caractère d'utilité publique de l'opération». Le Conseil d'État considère que le caractère extérieur et postérieur à l'acte de ce document exclut de l'assimiler à une véritable motivation en la forme qui serait une condition de la légalité de l'acte. Il s'agit de porter à la connaissance du public les motifs justifiant l'utilité publique et le Conseil d'État n'assimile pas cette obligation à une motivation en la forme ${ }^{43}$. Il a d'ailleurs jugé que le fait de ne pas prévoir

40. TA Paris, 18 juin 2004, Association Environnement $X V^{e}$, association Orbital et autres, L'actualité juridique. Droit administratif, 2005, p. 160, note P. Bon.

41. CE, 8 juillet 1994, Association des loueurs de scooters saintois, $\mathrm{n}^{\circ} 132295$, inédit au Recueil Lebon

42. Cass. crim., 20 novembre 2012, $\mathrm{n}^{\circ}$ 12-83.339, Droit pénal, $\mathrm{n}^{\circ}$ 2, février 2013, comm. 22, obs. J.-H. Robert.

43. CE, 25 avril 2007, Commune de Beauregard-de-Terrasson, $n^{\circ} 283016$, Bulletin juridique des collectivités locales, 2007, p. 569, concl. E. Prada-Bordenave. 
cette exigence de motivation ne méconnaît pas les droits garantis par l'article 7 de la Charte de l'environnement ${ }^{44}$. Pour autant, cette solution ne saurait mettre fin au débat sur l'intérêt de la motivation de la déclaration d'utilité publique. La motivation externe de la déclaration d'utilité publique joue une partie du rôle assigné à la motivation en la forme et révèle de manière très symbolique la recherche constante d'équilibre entre efficacité administrative et transparence. Elle éclaire d'un jour nouveau les enjeux spécifiques de la motivation des actes locaux, entre libre administration et intérêt public local.

\section{B. La motivation des actes locaux, une fonction ambivalente au regard de la libre administration et de l'intérêt public local}

Sans aucun doute, la transparence des procédures administratives se révèle un précieux auxiliaire pour leur compréhension, afin d'asseoir leur légitimité et la communication avec les administrés. Les choix faits parfois au nom de l'intérêt local par les représentants de la collectivité ne sauraient être davantage incontestables pour l'administré que ceux qui relèvent de la poursuite d'un intérêt strictement général. L'administré-citoyen entend légitimement connaître les raisons des décisions prises, exigeant naturellement de l'institution locale commune le respect du droit reconnu par la Déclaration des droits de l'homme dans son article 15, selon lequel «la société a le droit de demander compte à tout agent public de son administration ».

Ainsi, dans le cas des déclarations d'utilité publique concernant des opérations sensibles sur le plan environnemental, les revendications des administrés rejoignent les exigences du droit européen ${ }^{45}$ et international et celles de la Charte de l'environnement pour justifier une obligation de motivation. Si la loi du 27 février 2002 relative à la démocratie de proximité s'est limitée à prévoir que la déclaration d'utilité publique donnant lieu à enquête environnementale doit être accompagnée «d'un document qui expose les motifs et considérations justifiant son utilité publique» (art. L. 122-1 du nouveau Code de l'expropriation) plutôt que d'imposer une motivation en la forme, c'est à l'évidence pour ménager l'exigence de transparence tout en sécurisant l'acte administratif dont la validité ne sera ainsi pas remise en cause du fait d'un vice affectant le document annexe. Cela permet au juge administratif de préciser que

[...] les dispositions en cause du code de l'expropriation ne [peuvent] être interprétées comme imposant une motivation en la forme de la déclaration d'utilité publique qui serait une condition de légalité de cette dernière $[\ldots]^{46}$.
Ce faisant, le juge souligne tacitement le fait que la motivation n'élimine pas les risques de contentieux et peut même les accroître dans certaines hypothèses. Et ce constat dans la pratique locale apparaît déterminant, les multiples fonctions exercées par la motivation pouvant parfois s'entrechoquer. En justifiant de la rationalité des décisions administratives et en démontrant aux administrés que leur situation ou leurs demandes ont bien été prises en compte, l'administration peut éviter le contentieux. Mais une motivation inadaptée peut aussi faciliter ou susciter des recours.

Facteur de transparence de la décision administrative, la motivation peut aussi apparaître comme une limite à la libre administration des collectivités, principe constitutionnel qui inscrit l'action des collectivités dans le cadre défini par la loi, et reconnaît une liberté d'agir aux collectivités. Dans le domaine de l'emploi public local, par exemple, certains particularismes de la mise en œuvre du statut de la fonction publique sont très largement liés à une conception extensive de la libre administration soutenue par les relations de plus grande proximité au sein des collectivités. Les élus de celles-ci tendent à privilégier la plus grande liberté possible dans le recrutement et la gestion des collaborateurs. L'histoire de la fonction publique territoriale met en lumière une concurrence entre une conception modérée d'une libre administration encadrée, promue par le législateur, et une conception attractive du principe, souvent privilégiée par les autorités territoriales ${ }^{47}$. Une telle conception nourrit bien évidemment les réticences à la motivation des actes. Mais elle ne saurait occulter le fait que la libre administration, qui se traduit certes par l'exercice de compétences propres confiées aux organes délibérants, fait place aussi au contrôle assuré par le représentant de l'État. En prévision de ce contrôle, l'acte administratif local doit aussi intégrer la motivation, qui non seulement explicite la décision mais contribue au dialogue entre le contrôleur et le contrôlé. L'obligation de motiver contraint les autorités administratives à une analyse plus attentive du bien-fondé de leurs décisions: elle pourrait dès lors contribuer à une forme d'autocritique parfois bienvenue, lorsque la conception de la libre administration en vient à frôler l'arbitraire.

On ne saurait perdre de vue que les compétences confiées aux collectivités territoriales couvrent potentiellement tout le champ de l'activité administrative sous un angle opérationnel et suscitent le besoin de transparence et de motivation bien au-delà de la matière définie à l'article L. 211-2, englobant les règlements de police, la gestion des services publics et par là même les contrats. Les collectivités sont en outre aujourd'hui directement concernées par le droit d'accès aux données publiques ou l'open

44. CE, 19 avril 2013, Pernet Earl Pernet, nº 364498 , inédit au Recueil Lebon.

45. L'article 9 de la directive 97/11 CE du 3 mars 1997 dispose que les motifs et considérations ayant fondé les décisions prises sur certains projets publics ou privés doivent être mis à la disposition du public.

46. CE, 3 mars 2009, Syndicat national des industries d'information de santé (SNIS), nº 300570 , Recueil Lebon.

47. Dans sa décision no 83-168 DC du 20 janvier 1984, le Conseil constitutionnel avait jugé que le principe de libre administration englobait celui de la liberté de gestion par la collectivité de ses propres agents, et avait dégagé une liste de prérogatives nécessaires à l'existence de la libre administration. 
data qui les place dans l'obligation de rendre directement disponibles leurs données. Du fait de la répartition des compétences, les nécessités propres de la coordination entre personnes publiques territoriales, et entre celles-ci et les institutions étatiques, impliquent parfois des mécanismes procéduraux spécifiques, et accentuent le besoin d'information sur les motifs des actes. Elles doivent aujourd'hui, en outre, intégrer les préoccupations environnementales qui ont vocation aux termes de la Charte de l'environnement à associer l'ensemble des populations concernées à la décision ${ }^{48}$. La justification de l'intérêt public local, par construction fluctuant, peut ainsi amener à motiver des décisions qui ne sont pas expressément concernées par l'obligation de motivation. Ainsi, les décisions de subvention n'ont pas à être motivées ${ }^{49}$, toutefois, pour des raisons de transparence, la collectivité est souvent amenée à justifier sa décision par une motivation qui mettra en évidence la présence d'un intérêt public local ${ }^{50}$.

La relation de proximité au sein des collectivités favorise une demande de transparence et de participation plus forte chez les administrés locaux qui rejoint les recommandations des instances européennes. L'espace local plus en phase avec les réalités concrètes permet le développement de la concertation et de la consultation, entraînant dans son sillage l'exigence de motivation.

Pour toutes ces raisons, la généralisation de celle-ci semble se profiler mais il conviendrait d'en effectuer le bilan coût-avantages qui peut-être conduirait, afin d'éviter le développement du risque contentieux lourd de conséquences pour certains actes, à se limiter parfois à une motivation externe ou par référence. L'excès d'informations, parfois trop techniques ou mal maîtrisées, peut se révéler en effet contre-productif et nuire en définitive à l'objectif légitime de contrôle et d'évaluation de l'action locale. Le choix d'une motivation généralisée mais différenciée dans la forme, selon les catégories d'actes et les enjeux dont ils sont porteurs, peut sembler minimaliste sur le plan de la rigueur juridique mais serait néanmoins plus satisfaisant pour la démocratie administrative et la consolidation d'une citoyenneté locale.

48. Article 7 de la Charte de l'environnement: «Toute personne a le droit, dans les conditions et les limites définies par la loi, d'accéder aux informations relatives à l'environnement détenues par les autorités publiques et de participer à l'élaboration des décisions publiques ayant une incidence sur l'environnement ».

49. CE, 25 septembre 1995, Association connaître et informer pour valoriser l'importance des citoyens (CIVIC), $\mathrm{n}^{\circ} 155970$.

50. Voir les critères exigeant la présence d'un intérêt local et la neutralité politique posés par CE, 4 avril 2005, Commune d'Argentan, ${ }^{\circ}{ }^{264596}$, Recueil Lebon, p. 137. 